\section{Condições de vida e mortalidade infantil no Estado do Paraná, Brasil, 1997/2001}

\author{
Living conditions and infant mortality \\ in the state of Paraná, Brazil, 1997/2001
}

\author{
Selma Maffei de Andrade 1 \\ Darli Antonio Soares 1 \\ Tiemi Matsuo 2 \\ Regina Kazue Tanno de Souza 3 \\ Thaís Aidar de Freitas Mathias 3 \\ Maria Luiza Hiromi Iwakura 4 \\ Maria Angelina Zequim 4
}

\section{Introdução}

1 Centro de Ciências da Saúde, Universidade Estadual de Londrina, Londrina, Brasil.

2 Centro de Ciências Exatas, Universidade Estadual de

Londrina, Londrina, Brasil.

3 Centro de Ciências

da Saúde, Universidade

Estadual de Maringá,

Maringá, Brasil.

4 Diretoria de Processamento

e Análise de Dados em

Saúde, Autarquia Municipal

de Saúde de Londrina,

Londrina, Brasil.

Correspondência

S. M. Andrade

Departamento de Saúde

Coletiva, Centro de Ciências

da Saúde, Universidade

Estadual de Londrina.

Rua Pernambuco 1227

apto. 204, Londrina, $P R$

86020-121, Brasil.

semaffei@sercomtel.com.br

\section{Abstract}

This objective of this paper was to verify the relations between the living and infrastructure conditions of municipalities in the State of Paraná, Brazil, and infant mortality rate and its components. An ecological study was carried out having the 399 municipalities of the State being classified into five clusters of living conditions. Infant, neonatal and postneonatal rates were calculated for the period 1997/2001. The mean infant, neonatal and postneonatal mortality rates in the State were, respectively, 19.3, 12.0 and 7.3 per 1,000 live births. Postneonatal mortality rates ranged from 8.7 (cluster of worst living conditions) to 6.3 per 1,000 live births (in the cluster classified as having the best living conditions). Regarding the neonatal component, clusters classified as holding the best and the worst living conditions presented the lowest values: 11.8 and 11.4 per 1,000 live births, respectively. We conclude that the postneonatal component still adequately discriminate living conditions in the State of Paraná. However, there is a need of complementary studies that aim to clarify the causes of similar neonatal mortality rates observed in the richest and in the poorest cluster of municipalities.

Infant Mortality; Postneonatal Mortality; Living Conditions
Pela vulnerabilidade às condições de vida e de acesso a bens e serviços, o primeiro ano de vida constitui-se em um dos períodos de maior risco de morte. Assim, a taxa de mortalidade infantil (TMI) tem sido considerada um indicador sensível às condições sociais e de saúde das populações humanas 1 .

Nas últimas décadas, entretanto, mesmo em países de economia dependente ou onde persistem enormes desigualdades sociais ou, ainda, quando ocorrem períodos de crise econômica, tem-se observado decréscimo no valor dessa taxa, o que tem sido atribuído, principalmente, à redução da fecundidade e à expansão de serviços de saúde e de outros benefícios sociais, além de maior acesso a novas tecnologias em saúde 2,3,4,5,6.

No continente americano, também foi registrada notável redução da mortalidade infantil desde a segunda metade do século XX: de uma média de 90,34 óbitos de menores de um ano em cada mil nascidos vivos, nos anos 50 , para uma taxa média de 31,31 nos anos 90 . Apesar dessa redução, as desigualdades no risco de morte infantil persistiram em todo o período, com uma mortalidade cerca de quatro a cinco vezes maior nos países mais pobres, em comparação aos mais ricos 5 . Além disso, na América Latina, com o agravamento da crise econômica em meados da década de 1980, e sua 
persistência na década seguinte, observou-se aumento da pobreza e da concentração de renda em várias nações, com conseqüente redução da intensidade de declínio do coeficiente de mortalidade infantil na região 7 .

No Brasil, de 1991 a 1999, foi descrita uma redução de $28 \%$ no valor da TMI, atingindo uma média de 31,8 por mil nascidos vivos no último ano analisado 8 . Em 2000, a taxa estimada pelo Ministério da Saúde (MS) era de 28,3 por mil nascidos vivos 9 , superior, segundo dados do Centro Latino-americano de Perinatologia e Desenvolvimento Humano (CLAP) 10, à taxa apresentada por diversos países sul-americanos, em anos próximos, tais como Argentina (16,6 em 2000), Chile (10,1 em 1999), Paraguai (19,4 em 1999), Uruguai (14,1 em 2000) e Venezuela (17,7 em 2000).

A Região Sul do Brasil, composta pelos Estados do Rio Grande do Sul, Santa Catarina e Paraná, ainda que apresente o melhor desempenho em relação à mortalidade infantil no país 8,9 , carece de estudos que avaliem diferenciais nos padrões dessa mortalidade segundo condições de vida.

De acordo com o MS, o Paraná é o Estado da Região Sul que apresenta valores mais elevados da TMI (19,6 por mil nascidos vivos em 2000), em comparação a 15,1 no Estado do Rio Grande do Sul e 15,9 em Santa Catarina 9. Conhecer os diferenciais nos padrões dessa mortalidade, de acordo com as condições de vida e ambiente das comunidades, é fundamental para o estabelecimento de políticas públicas mais apropriadas a cada realidade. Nesse sentido, o presente trabalho teve como objetivo verificar se as condições de vida e de infra-estrutura dos municípios do Paraná se relacionam com a taxa de mortalidade infantil e seus componentes.

\section{Material e método}

Foi realizado um estudo de agregados, tendo como unidades iniciais de análise os $399 \mathrm{mu}$ nicípios do Paraná, o qual, segundo o Censo Demográfico de 2000, contava com uma população total de cerca de 9,5 milhões de habitantes 11. Posteriormente, esses municípios foram agrupados segundo condições de vida em cinco estratos (clusters) de acordo com as condições das famílias e de infra-estrutura dos municípios.

Essa classificação baseou-se, preliminarmente, na análise de dez variáveis derivadas do Censo Demográfico de 2000: (a) proporção de domicílios particulares permanentes cujos responsáveis não têm rendimento ou recebem menos que dois salários mínimos; (b) rendimento médio nominal mensal das pessoas responsáveis por domicílios particulares permanentes, entre os com rendimento; (c) rendimento mediano nominal mensal das pessoas responsáveis por domicílios particulares permanentes, entre os com rendimento; (d) proporção de domicílios particulares permanentes sem banheiro ou sanitário; (e) proporção de domicílios particulares permanentes com coleta de lixo; (f) proporção de domicílios particulares permanentes com abastecimento de água da rede geral; (g) proporção de responsáveis por domicílios particulares permanentes sem instrução ou com menos de oito anos de estudo; (h) proporção da população residente vivendo em domicílios improvisados; (i) proporção da população com dez anos ou mais de idade alfabetizada; (j) taxa de urbanização. Entre as três variáveis relacionadas à renda, optou-se por trabalhar apenas com a variável “proporção de domicílios particulares permanentes cujos responsáveis não têm rendimento ou recebem menos que dois salários mínimos", primeiro porque essa apresentava alta correlação com as duas outras (renda média e mediana mensal dos responsáveis por domicílios, entre os com rendimento) e, em segundo lugar, porque, na opinião dos autores, a variável selecionada traduziria melhor as condições sociais dos municípios, já que englobava a parcela de responsáveis por domicílios sem quaisquer rendimentos. Assim, permaneceram, na análise, oito variáveis.

Foi realizada, então, análise de componentes principais com o intuito de selecionar um número menor de variáveis 12 que refletissem as condições sociais dos municípios paranaenses. Do primeiro componente, com autovalor de 6,073 e explicando $60,73 \%$ da variância total, foram selecionadas as três variáveis que apresentaram maior correlação com este componente (coeficiente de saturação $\geq 0,91$ ): (a) proporção de domicílios particulares permanentes com abastecimento de água da rede geral, (b) proporção de domicílios particulares permanentes com coleta de lixo e (c) taxa de urbanização. Da mesma forma, do segundo componente, com autovalor de 1,593 e explicando 15,93\% da variância total, foram selecionadas as duas variáveis mais correlacionadas (coeficiente de saturação $\geq 0,84$ : (a) proporção de domicílios particulares permanentes cujos responsáveis não têm rendimento ou recebem menos que dois salários mínimos e (b) proporção da população com dez anos ou mais de idade alfabetizada. As variáveis selecionadas do primeiro componente (urbanização, coleta 
de lixo e abastecimento de água) refletem as condições de infra-estrutura dos municípios, enquanto as do segundo (escolaridade e renda), as condições de vida das famílias.

Para cada uma dessas cinco variáveis selecionadas pela análise de componentes principais, os municípios foram ordenados da pior para a melhor situação, recebendo pontos correspondentes ao posto obtido para cada uma das variáveis, sendo, então, feito o somatório desses pontos, com definição do respectivo escore. O município com piores condições de vida, teoricamente, receberia um escore de cinco pontos (um ponto referente a cada uma das variáveis) e o com melhor condição, 1.995 pontos (todas as cinco variáveis no 399o posto). Na prática, a pontuação variou de 58 a 1.993 .

Após ordenação geral desses municípios por escores, foram feitos agrupamentos dos municípios por quintis e por clusters por meio do método não-hierárquico $k$-means, o qual também gerou cinco estratos. Para cada um dos dois tipos de agrupamentos (por quintis ou clusters), realizou-se análise de variância e aplicouse o teste de Tukey post-hoc 13, para comparação das médias de cada uma das variáveis que compunham o escore, sendo o agrupamento por clusters considerado o de maior poder discriminatório das condições sociais dos municípios do Paraná. Esse tipo de análise resultou, portanto, em cinco estratos de municípios, ordenados, segundo condições de vida, da pior (cluster 1) para a melhor situação (cluster 5).

Com o objetivo de averiguar se a classificação final dos municípios nos cinco clusters refletia adequadamente as condições de vida dos municípios, esses clusters foram comparados, ainda, ao Índice de Desenvolvimento Humano Municipal (IDH-M) do Paraná 14. Essa análise indicou que os clusters construídos estiveram associados a esse indicador de desenvolvimento humano, pois se observou aumento do IDH$\mathrm{M}$ dos clusters à medida que estes eram considerados como de melhor condição de vida, com diferenças significantes do IDH-M médio e dos componentes "educação" e "renda" entre todos os clusters. O componente "longevidade" da população, que também compõe o IDH-M, embora tenha apresentado elevação de valores com a melhoria da classificação do cluster, apresentou sobreposição dos intervalos de confiança de 95\% (IC95\%) entre clusters consecutivos ( 1 e 2, 2 e 3, 3 e 4, 4 e 5). Assim, considerou-se que a classificação dos cinco clusters, no presente estudo, discriminaria adequadamente as condições sócio-econômicas dos municípios paranaenses 14. Optou-se por trabalhar com essa classificação de cluster, e não com o IDH-M, por esse incluir, entre seus componentes, a "esperança de vida ao nascer”, a qual é influenciada pela taxa de mortalidade infantil, foco deste trabalho.

Para o cálculo da taxa de mortalidade infantil e de seus componentes (neonatal - até 28 dias - e pós-neonatal - de 28 dias até um ano), foram obtidos dados sobre óbitos de menores de um ano e de nascidos vivos da página eletrônica da Secretaria de Estado da Saúde do Paraná 15 para o qüinqüênio 1997/2001, a fim de aumentar a estabilidade das taxas. Os valores absolutos foram transportados para planilha do programa Excel for Windows, sendo então calculadas as taxas qüinqüenais para cada cluster.

Após análise da mortalidade geral padronizada por idade, tendo como referência o padrão etário da população do Paraná, foram excluídos do estudo 14 municípios, os quais, possivelmente, apresentavam problemas de cobertura da informação sobre mortalidade, por terem registrado um coeficiente de mortalidade geral padronizado por idade menor do que 4,0 por mil habitantes, no triênio 1999/2001 16 .

Para verificar a possibilidade de classificação errônea de óbitos neonatais precoces (menores de sete dias) como óbitos fetais, analisaram-se também as diferenças em relação às taxas de natimortalidade nesses clusters de municípios (óbitos de fetos com 500g ou mais, ou com duração da gestação $\geq 22$ semanas, em relação ao total de nascimentos).

O programa SAS (SAS Institute Inc., Cary, Estados Unidos) foi utilizado nas análises estatísticas. Os resultados foram apresentados em taxas e com seus respectivos IC95\%.

\section{Resultados}

As principais características dos cinco clusters de municípios segundo condições de vida são apresentadas na Tabela 1, a qual mostra melhoria dos indicadores à medida que o cluster é classificado em nível imediatamente superior. Com exceção do cluster 5 (de melhor situação), os demais apresentam mais de $60 \%$ dos responsáveis pela família com rendimento máximo de dois salários mínimos, chegando a praticamente $80 \%$ no primeiro cluster. Com relação ao abastecimento de água, apenas dois clusters (o 4 e o 5) possuem, em média, mais de $75 \%$ dos domicílios com acesso à água encanada no domicílio. A taxa de alfabetização entre pessoas com dez anos ou mais foi a variável que apresentou menor variabilidade entre os clusters, sendo, porém, superior a $90 \%$ apenas 
Clusters de municípios segundo condições de vida de acordo com o número de domicílios, habitantes e variáveis analisadas (proporção e intervalo de confiança de 95\% - IC95\%). Paraná, Brasil, 2000.

\begin{tabular}{|c|c|c|c|c|c|c|c|c|c|c|c|c|}
\hline \multirow[t]{2}{*}{ Cluster } & \multirow[t]{2}{*}{$\begin{array}{l}\text { Domi- } \\
\text { cílios }\end{array}$} & \multirow[t]{2}{*}{$\begin{array}{l}\text { Popu- } \\
\text { lação }\end{array}$} & \multicolumn{2}{|c|}{$\begin{array}{l}\text { Responsáveis } \\
\text { pelos domicílios } \\
\text { com renda }<2 \\
\text { salários mínimos }\end{array}$} & \multicolumn{2}{|c|}{$\begin{array}{l}\text { Domicílios ligados } \\
\text { à rede de água }\end{array}$} & \multicolumn{2}{|c|}{$\begin{array}{l}\text { Domicílios com } \\
\text { coleta de lixo }\end{array}$} & \multicolumn{2}{|c|}{ 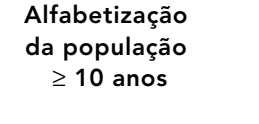 } & \multicolumn{2}{|c|}{$\begin{array}{c}\text { Taxa de } \\
\text { urbanização }\end{array}$} \\
\hline & & & $\%$ & IC95\% & $\%$ & IC95\% & $\%$ & IC95\% & $\%$ & IC95\% & $\%$ & IC95\% \\
\hline 1 & 95.910 & 381.245 & 78,61 & $78,35-78,87$ & 39,70 & $39,39-40,01$ & 32,06 & $31,77-32,36$ & 81,79 & $81,65-81,93$ & 29,31 & $29,17-29,46$ \\
\hline 2 & 213.487 & 809.022 & 71,82 & $71,63-72,01$ & 54,52 & $54,31-54,73$ & 47,75 & $47,54-47,96$ & 85,88 & $85,79-85,96$ & 44,86 & $44,75-44,97$ \\
\hline 3 & 256.075 & 936.555 & 66,55 & $66,37-66,73$ & 67,77 & $67,59-67,95$ & 63,16 & $62,97-63,35$ & 87,53 & $87,45-87,60$ & 61,87 & $61,77-61,97$ \\
\hline 4 & 352.525 & 1.273 .643 & 60,06 & $59,90-60,23$ & 78,44 & $78,30-78,58$ & 77,11 & $76,97-77,25$ & 88,59 & $88,52-88,65$ & 71,23 & $71,15-71,31$ \\
\hline 5 & 1.746 .279 & 6.162 .993 & 38,66 & $38,59-38,73$ & 92,96 & $92,92-92,99$ & 94,54 & $94,50-94,57$ & 93,87 & $93,85-93,89$ & 94,51 & $94,49-94,53$ \\
\hline
\end{tabular}

no cluster 5 . Todos os municípios considerados pólos regionais, com mais de 80 mil habitantes, foram classificados no cluster 5 , de melhores condições de vida. O número de habitantes dos municípios foi crescente nos clusters, com média (e desvio padrão) de $7.943(5.253,2), 8.890$ (6.752,3), 9.756 (7.401,3), $14.152(12.621,0) \mathrm{e}$ $83.284(194.218,5)$ nos clusters $1,2,3$, 4 e 5, respectivamente.

No qüinqüênio 1997/2001, no Estado do Paraná, os sistemas de informações vitais registraram 911.799 nascidos vivos, 11.188 óbitos neonatais e 6.374 óbitos pós-neonatais, representando taxas médias de mortalidade infantil, neonatal e pós-neonatal, respectivamente, de 19,3, 12,3 e 7,0 por mil nascidos vivos.

A análise e exclusão dos 14 municípios nos quais a cobertura da informação sobre mortalidade poderia ser inadequada revelaram que esses se concentraram no cluster de piores condições de vida (cluster 1), com a seguinte distribuição e proporcionalidade em relação ao tamanho de cada cluster: seis municípios do cluster $1(12,5 \%)$, dois do cluster $2(2,2 \%)$, cinco do cluster $3(5,2 \%)$ e um do cluster $4(1,1 \%)$. Não houve município excluído do cluster de melhores condições de vida (cluster 5 ).

A Tabela 2 mostra as taxas qüinqüenais da mortalidade infantil e de seus componentes para os cinco clusters de municípios do Estado. Em primeiro lugar, observa-se que o componente neonatal da mortalidade infantil predominou em todos os clusters, variando de um menor valor no cluster de piores condições de vida (11,4 por mil nascidos vivos) até um máximo de 13,7 no cluster intermediário (cluster 3 ). Para esse componente, notam-se taxas semelhantes de mortalidade e sobreposição dos IC95\% dessas taxas dos clusters 1 e 5 , sendo es- tas menores do que as dos clusters intermediários (clusters 2, 3 e 4).

Com relação ao componente pós-neonatal, não obstante a tendência de decréscimo desse valor de acordo com a classificação dos clusters por condições de vida, apenas no cluster de melhores condições de vida (cluster 5 ) foi observada uma taxa que não apresentava sobreposição dos IC95\% com as dos demais.

Com base no resultado observado da taxa de mortalidade neonatal (menor no cluster de piores condições de vida), decidiu-se avaliar a possibilidade de classificação errônea de mortes neonatais precoces (antes dos sete dias de vida) como óbitos fetais, o que inflaria a taxa de natimortalidade e reduziria, artificialmente, a taxa de mortalidade neonatal. Como se pode observar na Figura 1, esse indicador apresentou um padrão bastante semelhante ao do componente neonatal da TMI e também foi menor no cluster de piores condições de vida $(11,3$ por mil nascimentos), e semelhante à taxa do cluster de melhores condições (11,6 por mil).

\section{Discussão}

Em comparação ao componente neonatal da mortalidade infantil, o pós-neonatal tem sido considerado um indicador mais específico das condições de vida, em geral, pelo padrão das causas de morte que prevalecem nesse período (doenças infecciosas e parasitárias, doenças do aparelho respiratório, entre outras) 1 . Não obstante esse perfil tenha se alterado em anos recentes, especialmente em grandes e médios centros urbanos, em razão da expansão das unidades de terapia intensiva e de novas práticas assistenciais neonatais, com conseqüente pro- 
longamento da sobrevida de recém-nascidos, e aumento da mortalidade por afecções perinatais após o primeiro mês de vida 17,18 , no presente estudo, observou-se que esse componente apresentou, como esperado, um padrão relacionado às condições de vida dos agregados de municípios, com decréscimo do valor da taxa à medida que o cluster se situava em um melhor nível. Há que se destacar, no entanto, que apenas o cluster considerado como de melhores condições de vida (cluster 5) apresentou uma taxa cujos valores do IC95\% não se sobrepunham aos intervalos de confiança das demais.

Nos últimos anos, no Brasil, tem sido registrada maior redução da mortalidade infantil, especialmente do componente pós-neonatal, em áreas com condições sociais mais desfavoráveis, queda essa atribuída, sobretudo, aos valores iniciais mais elevados do que os apresentados por áreas mais ricas, além dos efeitos da expansão de serviços de saúde e da transferência de tecnologias, propiciando acesso mais amplo a esses recursos, inclusive a parcelas da população menos privilegiadas socialmente 2,3,4,5,19.

Assim, observa-se, atualmente, certa tendência de homogeneização das taxas de mortalidade infantil entre áreas ricas e pobres 3 , o que tem levado alguns pesquisadores a afirmar que não é suficiente levar em consideração apenas os determinantes sócio-econômicos nesse desfecho, mas também os efeitos dos serviços de saúde e de como esses podem atuar na intermediação entre condições de vida e a situação de saúde 20 . No presente trabalho, embora não se tenha levado em consideração a atuação desses serviços, o achado relativo ao comportamento do componente pós-neonatal reforça a tese de que esse é, ainda, um indicador sensível às desigualdades nas condições de vida das populações 21 .

O quadro observado em relação ao componente neonatal, intrigante no sentido de apresentar valores semelhantes em estratos de municípios de piores e melhores condições de vida, estimula algumas reflexões.

Em primeiro lugar, é possível que diferenças entre as áreas, de acordo com as condições de vida, na realidade, existam, mas que não tenham sido detectadas pelo presente estudo. Um dos motivos para esse fato seria a existência de taxas de sub-registro de óbitos infantis precoces diferenciadas segundo os clusters de condições de vida, sendo esse sub-registro mais elevado em municípios de pior situação, conforme apontam outros estudos 3,22.

Apesar de o Estado do Paraná ter registrado importantes avanços na qualidade e na cobertura dos dados oficiais em saúde na última dé-
Tabela 2

Taxas de mortalidade neonatal, pós-neonatal e infantil (TMI) no qüinqüênio 1997/2001 e respectivos intervalos de confiança de 95\% (IC95\%) segundo clusters de condições de vida no Estado do Paraná, Brasil.

\begin{tabular}{lcccccc}
\hline \multirow{2}{*}{ Cluster } & \multicolumn{2}{c}{ Neonatal } & \multicolumn{2}{c}{ Pós-neonatal } & \multicolumn{2}{c}{ TMI } \\
& Taxa & IC95\% & Taxa & IC95\% & Taxa & IC95\% \\
\hline 1 & 11,4 & $10,4-12,5$ & 8,7 & $7,8-9,7$ & 20,1 & $18,8-21,6$ \\
2 & 13,4 & $12,6-14,3$ & 8,6 & $8,0-9,3$ & 22,1 & $21,0-23,1$ \\
3 & 13,7 & $13,0-14,6$ & 8,4 & $7,8-9,0$ & 22,1 & $21,2-23,2$ \\
4 & 13,2 & $12,6-13,9$ & 7,9 & $7,4-8,4$ & 21,1 & $20,3-21,9$ \\
5 & 11,8 & $11,6-12,1$ & 6,3 & $6,1-6,5$ & 18,2 & $17,8-18,5$ \\
\hline
\end{tabular}

Figura 1

Taxa de natimortalidade no qüinqüênio 1997/2001 e respectivos intervalos de confiança de 95\% (IC95\%) segundo clusters de condições de vida no Estado do Paraná, Brasil.

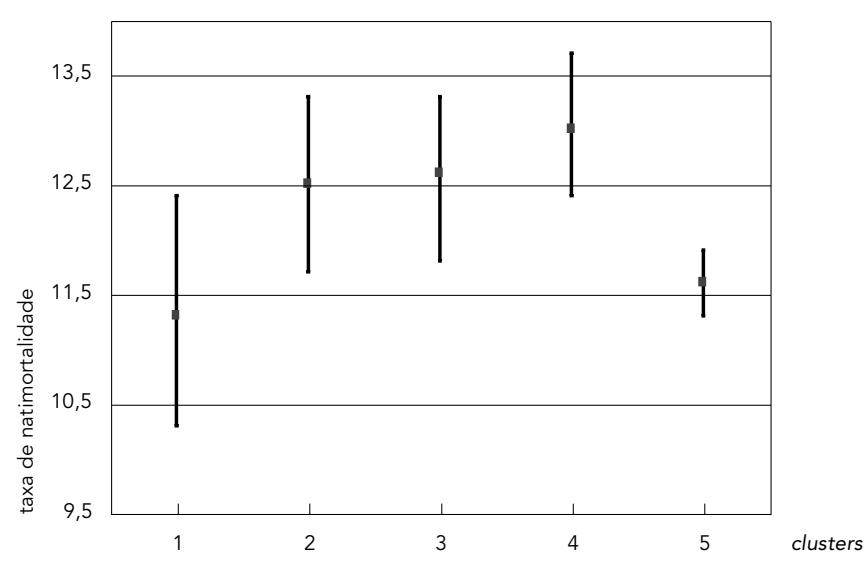

cada, com a municipalização dos serviços de saúde e a busca ativa de informações sobre nascimentos e óbitos 23,24 , esse avanço ocorreu principalmente em médias e grandes cidades. Assim, é provável que o problema persista em grande parte dos municípios de pequeno porte, os quais predominam nos estratos de piores condições de vida. Essa possibilidade é reforçada quando se analisam os 14 municípios excluídos da análise deste trabalho, por apresentarem um coeficiente geral de mortalidade padronizado por idade menor do que 4,0 por mil habitantes: seis $(42,9 \%)$ pertenciam ao cluster de piores condições de vida. Além disso, esses municípios representaram $12,5 \%$ do total de 
municípios do cluster 1 , sendo esta a maior proporção de municípios excluídos de um cluster, o que indica piores coberturas da informação sobre mortalidade 16. Por outro lado, não houve municípios com coeficiente geral de mortalidade padronizado por idade menor do que 4 por mil habitantes no cluster de melhores condições de vida.

Embora o problema da análise das taxas pudesse ter sido parcialmente contornado por meio do uso de estimativas de taxas de mortalidade infantil 16, no presente estudo, optou-se pela utilização dos dados oficiais do Ministério da Saúde, por dois motivos principais: primeiro, por ter sido observado que as estimativas indiretas do Instituto Brasileiro de Geografia e Estatística (IBGE) 25 apresentavam valores superiores aos oficiais (cálculo direto), mesmo em municípios paranaenses com alta cobertura e qualidade da informação sobre mortalidade infantil, fato que corrobora a hipótese de que as estimativas indiretas nem sempre refletem a realidade 16,26. Em segundo lugar, entende-se que a utilização de dados oficiais na análise da situação de saúde pode contribuir para o processo em curso no Brasil de qualificação da informação em saúde.

O sub-registro de óbitos infantis pode ser ocasionado tanto pela não emissão da declaração de óbito (por existência de cemitérios clandestinos ou de cemitérios que não exijam esse documento para o enterramento, por exemplo), como pela classificação errônea de óbitos neonatais precoces como óbitos fetais, especialmente aqueles que ocorrem nos primeiros minutos após o nascimento, fato que reduz de forma artificial, especificamente, o componente da mortalidade infantil neonatal precoce (até sete dias de vida) e aumenta, também artificialmente, a taxa de natimortalidade, porém que não altera a taxa de mortalidade perinatal 27 . No presente estudo, o padrão das taxas de natimortalidade, todavia, foi bastante semelhante ao das taxas de mortalidade neonatal, o que sugere que a classificação errônea de óbitos neonatais como óbitos fetais, caso exista, não seja um problema exclusivo do cluster de piores condições de vida, mas similar em todos os clusters.

Dessa forma, a existência de sub-registro de óbitos infantis por problemas de omissão ou fluxo das declarações de óbito nos municípios de piores condições de vida é, aparentemente, uma suposição mais plausível. Assim, há necessidade de se investigar a existência de cemitérios que não exijam a declaração de óbito para o enterramento nesses municípios, especialmente de crianças que morrem ao nas- cer ou durante a gestação. Além disso, há possibilidade, ainda, de existência de dificuldades relacionadas ao fluxo das declarações de óbito, ou seja, a não captação, pelo Sistema de Informação em Mortalidade (SIM), de declarações de óbito corretamente emitidas. Esse fluxo pode apresentar problemas diversos, como, por exemplo, extravio dos documentos ou armazenamento indevido, fazendo com que o documento não chegue ao conhecimento do sistema de informação do Ministério da Saúde.

Além dessas hipóteses, migração de óbitos, com óbitos de residentes em áreas de piores condições de vida sendo registrados como residentes em áreas de melhores condições, por ocorrência do óbito nessas últimas quando em busca de assistência médica, também tem sido uma possibilidade aventada em pesquisas semelhantes 22,28 para explicar reduções ou aumentos artificiais da taxa de mortalidade infantil. Essas várias hipóteses sugerem a necessidade de estudos adicionais, que avaliem a qualidade, a cobertura e o fluxo da informação sobre eventos vitais nos municípios que, apesar das piores condições de vida, apresentaram menores taxas de mortalidade neonatal e de natimortalidade.

Por outro lado, uma segunda reflexão, a respeito das taxas semelhantes de natimortalidade e de mortalidade neonatal nos clusters de melhores e de piores condições de vida, não pode deixar de ser exposta. É também admissível que os valores sejam realmente semelhantes, porém com características diversas. Sabese que a cobertura e qualidade da assistência médica e hospitalar nos municípios de maior porte (concentrados no cluster de melhores condições) possibilita maior acesso a técnicas de reprodução assistida, o que tem gerado inúmeras gestações múltiplas, com aumento da mortalidade fetal e de nascidos vivos que, muitas vezes, não sobrevivem ao primeiro mês de vida 17 . Por outro lado, nos municípios de menor porte, os óbitos neonatais e fetais podem estar mais relacionados às maiores dificuldades de acesso e às características da atenção à gestação e ao parto. Investigações que avaliem o perfil dessa mortalidade nos diferentes estratos de municípios, portanto, poderiam colaborar no direcionamento de ações específicas que visem a reduzi-la.

Em relação ao comportamento do componente neonatal da mortalidade infantil, finalmente, há que se destacar que o tipo de estudo realizado, de agregados (ou ecológico), possui limitações inerentes ao seu desenho. Por trabalhar com taxas médias baseadas em grupos, não é possível associar exposição e desfecho 
em nível individual, o que pode levar a hipóteses equivocadas, fenômeno conhecido como "falácia ecológica" 29,30. Uma forma de se reduzir esse viés é se trabalhar com áreas geográficas pequenas, visando torná-las o mais homogêneas possível, o que gera, porém, outros problemas, tais como a redução da estabilidade das taxas 29. No presente estudo, apesar das técnicas utilizadas no sentido de agrupar municípios em estratos homogêneos de condições de vida, a heterogeneidade intra-estratos pode ter contribuído para os resultados encontrados. Residentes em municípios de piores condições de vida que se localizam próximos a centros urbanos maiores têm, por exemplo, maior possibilidade de acesso a recursos humanos e tecnológicos de saúde do que residentes em municípios classificados no mesmo estrato de condições de vida, porém mais distantes. Por outro lado, em municípios maiores, os bolsões de pobreza podem contribuir para elevar a média das taxas de mortalidade.

Apesar dessas limitações, cabe ressaltar que o objetivo deste trabalho não foi o de determinar riscos à mortalidade infantil em nível individual, mas o de descrever os riscos desse tipo de mortalidade relacionados ao contexto social e de infra-estrutura dos municípios do Paraná, com vistas a identificar agregados de sobre-risco 31 , passíveis de intervenção por meio de po- líticas setoriais ou intersetoriais 31,32 . Nesse sentido, a abordagem feita por estudos ecológicos torna-se mais apropriada $31,32,33$.

Tendo em mente essas considerações, o presente trabalho revelou que o componente pós-neonatal da mortalidade infantil ainda discrimina adequadamente as condições de vida no Paraná. Com relação ao componente neonatal, há necessidade de outros estudos, em especial, os de base individual, que investiguem as características particulares dessa mortalidade nos diferentes estratos de condições de vida no Estado e o papel que os serviços de saúde, ao lado dessas condições de vida, têm na determinação de sua ocorrência. Nesse sentido, a expansão do trabalho dos Comitês de Prevenção da Mortalidade Infantil do Paraná, já implantados em cerca de $40 \%$ dos municípios paranaenses e com investigação de cerca de metade dos óbitos infantis ocorridos 24 , pode ser uma estratégia relevante para proporcionar um melhor conhecimento dessa realidade e contribuir para a adoção de ações que promovam a redução da mortalidade infantil evitável, ainda que não se descarte a preocupação com a necessidade de políticas sociais e econômicas mais amplas, que visem, em última instância, à redução das desigualdades sociais no Estado e no país como um todo.

\section{Resumo}

Com o objetivo de verificar se as condições de vida e de infra-estrutura dos municípios do Estado do Paraná, Brasil, se relacionam com a taxa de mortalidade infantil e seus componentes, foi realizado estudo de agregados, com os 399 municípios do Estado agrupados em cinco clusters de condições de vida. As taxas de mortalidade infantil, neonatal e pós-neonatal foram calculadas para o qüinqüênio 1997/2001. Observaram-se taxas médias de mortalidade infantil, neonatal e pós-neonatal no Estado de 19,3, 12,0 e 7,3 por mil nascidos vivos, respectivamente. A taxa de mortalidade infantil pós-neonatal variou de 8,7 (cluster de piores condições) a 6,3 por mil nascidos vivos (cluster de melhores condições de vida). Com relação ao compo- nente neonatal, os clusters de melhor e pior situação apresentaram as menores taxas, com valores semelhantes: 11,8 e 11,4 por mil nascidos vivos, respectivamente. Conclui-se que o componente pós-neonatal ainda discrimina adequadamente as condições de vida no Estado do Paraná, porém há necessidade de estudos complementares visando esclarecer as causas das taxas semelhantes de mortalidade neonatal dos clusters classificados como de melhores e de piores condições de vida.

Mortalidade Infantil; Mortalidade Pós-neonatal; Condições de Vida 


\section{Colaboradores}

Todos os autores participaram da concepção e planejamento da pesquisa. S. M. Andrade coordenou os trabalhos, participou da tabulação dos dados e redigiu a primeira versão do artigo. M. L. H. Iwakura e M. A. Zequim participaram na tabulação dos dados. T. Matsuo foi responsável pela análise estatística. Todos os autores contribuíram para a versão final do artigo e o aprovaram.

\section{Referências}

1. Vermelho LL, Costa AJL, Kale PL. Indicadores de saúde. In: Medronho RA, organizador. Epidemiologia. São Paulo: Atheneu; 2002. p. 33-55.

2. Costa MCN, Mota ELA, Paim JS, Silva LMV, Teixeira MG, Mendes MC. Mortalidade infantil no Brasil em períodos recentes de crise econômica. Rev Saúde Pública 2003; 37:699-706.

3. Goldani MZ, Benatti R, Silva AAM, Bettiol H, Correa JCW, Tietzmann M, et al. Narrowing inequalities in infant mortality in Southern Brazil. Rev Saúde Pública 2002; 36:478-83.

4. Rutstein SO. Factors associated with trends in infant and child mortality in developing countries during the 1990s. Bull World Health Organ 2000; 78:1256-70.

5. Schneider MC, Castillo-Salgado C, Loyola-Elizondo E, Bacallao J, Mujica OJ, Vidaurre M, et al. Trends in infant mortality inequalities in the Americas: 1955-1995. J Epidemiol Community Health 2002; 56:538-41.

6. Monteiro CA, Benício MHD’A, Freitas ICM. Evolução da mortalidade infantil e do retardo do crescimento nos anos 90: causas e impacto sobre desigualdades regionais. In: Monteiro CA, organizador. Velhos e novos males da saúde no Brasil. 2a Ed. São Paulo: Editora Hucitec/Núcleo de Pesquisas Epidemiológicas em Nutrição e Saúde, Universidade de São Paulo; 2000. p. 393-420.

7. Romero DE, Szwarcwald CL. Crisis económica y mortalidad infantil en Latinoamérica desde los años ochenta. Cad Saúde Pública 2000; 16:799814.

8. Duarte EC, Schneider MC, Paes-Souza R, Silva JB, Castillo-Salgado C. Expectativa de vida ao nascer e mortalidade no Brasil em 1999: análise exploratória dos diferenciais regionais. Rev Panam Salud Pública 2002; 12:436-44.

9. Departamento de Informática do SUS. Rede interagencial de informações para a saúde. Indicadores e dados básicos para a saúde - IDB 2002. http://tabnet.datasus.gov.br/cgi/idb2002/c01.ht $\mathrm{m}$ (acessado em 07/Jun/2004).

\section{Agradecimentos}

Ao Conselho Nacional de Desenvolvimento Científico e Tecnológico (CNPq) e à Fundação Araucária, pelo apoio financeiro (processo 521030/99-4 - Plano Sul de Pesquisa e Pós-graduação).

10. Centro Latinoamericano de Perinatología y Desarrollo Humano. Mortalidad materna-perinatalinfantil (América Latina y Caribe). http://www. paho.org/spanish/clap/05mort.htm (acessado em 09/Jul/2004).

11. Departamento de Informática do SUS. População residente - Brasil, 2000. http://tabnet.datasus. gov.br/cgi/tabcgi.exe?ibge/cnv/popuf.def (acessado em 05/Mar/2004).

12. Carvalho MS, Cruz OG, Nobre FF. Perfil de risco: método multivariado de classificação sócio-econômica de microáreas urbanas - os setores censitários da região metropolitana do Rio de Janeiro. Cad Saúde Pública 1997; 13:635-45.

13. Wayne DW. Biostatistics: a foundation for analysis in the health sciences. $6^{\text {th }}$ Ed. New York: John Wiley; 1995.

14. Andrade SM, Matsuo T, Soares D, Souza RKT, Mathias TAF, Iwakura MLH, et al. Condições de vida no Estado do Paraná: análise ecológica com base em variáveis do Censo Demográfico de 2000. Semina Cienc Biol Saúde 2004; 25:73-80.

15. Secretaria de Estado da Saúde do Paraná. Mortalidade perinatal e infantil. http://www.saude.pr. gov.br/Estatisticas/infantil/index.html (acessado em 12/Dez/2002).

16. Szwarcwald CL, Leal MC, Andrade CLT, Souza Jr. PRB. Estimação da mortalidade infantil no Brasil: o que dizem as informações sobre óbitos e nascimentos do Ministério da Saúde? Cad Saúde Pública 2002; 18:1725-36.

17. Ramos MR, García MAN. Evolución de la mortalidad infantil, neonatal y postneonatal en Andalucía, 1975-1998. Rev Esp Salud Pública 2003; 77:363-71.

18. Woolbright LA. Postneonatal mortality in Alabama: why no progress in the 90s? Ann Epidemiol 2001; 11:208-12.

19. Holcman MM, Latorre MRDO, Santos JLF. Evolução da mortalidade infantil na região metropolitana de São Paulo, 1980-2000. Rev Saúde Pública 2004; 38:180-6. 
20. Szwarcwald CL, Bastos FI, Andrade CLT. Medidas de desigualdad en salud: la discusión de algunos aspectos metodológicos con una aplicación para la mortalidad neonatal en el Municipio de Rio de Janeiro, 2000. Cad Saúde Pública 2002; 18:959-70.

21. Goldani MZ, Barbieri MA, Bettiol H, Barbieri MR, Tomkins A. Infant mortality rates according to socioeconomic status in a Brazilian city. Rev Saúde Pública 2001; 35:256-61.

22. Costa MCN, Azi PA, Paim JS, Silva LMV. Mortalidade infantil e condições de vida: a reprodução das desigualdades sociais em saúde na década de 90. Cad Saúde Pública 2001; 17:555-67.

23. Silva SF, Sugmyama LCY, Oliveira MSM, Petris AJP, Andrade SM, Oliveira EA, et al. Sistema de informações: instrumento de planejamento para redução de desigualdades. In: Silva SF, organizador. A construção do SUS a partir do município. São Paulo: Editora Hucitec; 1996. p. 12-28.

24. Mansano NH, Mazza VA, Soares VMN, Araldi MAR, Cabral VLM. Comitês de prevenção da mortalidade infantil no Paraná, Brasil: implantação e operacionalização. Cad Saúde Pública 2004; 20 : 329-32.

25. Departamento de Informática do SUS. Coeficiente de mortalidade infantil por ano segundo município - Paraná, 1989 a 1998. http://tabnet. datasus.gov.br/cgi/mortinf/municpr.htm (acessado em 07/Nov/2004).
26. Ministério da Saúde. Saúde Brasil 2004: uma análise da situação de saúde. Brasília: Secretaria de Vigilância à Saúde, Ministério da Saúde; 2004.

27. Laurenti R, Mello-Jorge MHP, Lebrão ML, Gotlieb SLD. Estatísticas de saúde. 2a Ed. São Paulo: E.P.U.; 1987.

28. Campos TP, Carvalho MS, Barcellos CC. Mortalidade infantil no Rio de Janeiro, Brasil: áreas de risco e trajetória dos pacientes até os serviços de saúde. Rev Panam Salud Pública 2000; 8:164-70.

29. Medronho RA. Estudos ecológicos. In: Medronho RA, Carvalho DM, Bloch KV, Luiz RR, Werneck GL, editores. Epidemiologia. São Paulo: Atheneu; 2002. p. 191-8.

30. Koifman S, Koifman RJ, Meyer A. Human reproductive system disturbances and pesticide exposure in Brazil. Cad Saúde Pública 2002; 18:435-45.

31. Carvalho MS, Souza-Santos R. Análise de dados espaciais em saúde pública: métodos, problemas, perspectivas. Cad Saúde Pública 2005; 21:361-78.

32. Silva LMV, Paim JS, Costa MCN. Desigualdades na mortalidade, espaço e estratos sociais. Rev Saúde Pública 1999; 33:187-97.

33. Chor D, Faerstein E. Um enfoque epidemiológico da promoção da saúde: as idéias de Geoffrey Rose. Cad Saúde Pública 2000; 16:241-4.

Recebido em 16/Dez/2004

Versão final reapresentada em 15/Jun/2005

Aprovado em 14/Jul/2005 